Monatsschr Kinderheilkd 2020 1 168:1130-1131 https://doi.org/10.1007/s00112-020-01055-y Online publiziert: 17 . November 2020 (c) Springer Medizin Verlag $\mathrm{GmbH}$, ein Teil von Springer Nature 2020

\section{Redaktion}

F. Hoffmann, München

\author{
Philipp Jung ${ }^{1,2,3} \cdot$ Lukas Mileder $^{2,4} \cdot$ Florian Hoffmann $^{1,2,5} \cdot$ Ellen Heimberg ${ }^{1,2,6}$ \\ 'PAEDSIM e. V., Tübingen, Deutschland \\ ${ }^{2}$ Netzwerk Kindersimulation e. V., Tübingen, Deutschland \\ ${ }^{3}$ Klinik für Kinder- und Jugendmedizin, Universitätsklinikum Schleswig-Holstein, Campus Lübeck, Lübeck, \\ Deutschland \\ ${ }^{4}$ Medizinische Universität Graz, Universitätsklinik für Kinder- und Jugendheilkunde, Graz, Österreich \\ ${ }^{5}$ Ludwig-Maximilians-Universität, Campus Innenstadt, Kinderklinik und Kinderpoliklinik im Dr. von \\ Haunerschen Kinderspital, München, Deutschland \\ ${ }^{6}$ Klinik für Kinderheilkunde und Jugendmedizin, Universitätsklinikum Tübingen, Tübingen, Deutschland
}

\title{
Simulationsbasierte pädiatrische Notfallteamtrainings in Zeiten der SARS-CoV-2-Pandemie
}

\section{Stellungnahme des Netzwerk Kindersimulation e. V. und des PAEDSIM e.V.}

Die bestmögliche Bewältigung eines medizinischen Notfalls ist von vielen Faktoren abhängig, v. a. aber auch von der guten und effektiven Zusammenarbeit des versorgenden Behandlungsteams [1]. Für Kindernotfälle gilt dies im Speziellen, da sie weniger häufig als vergleichbare Notfallsituationen in der Erwachsenenmedizin sind [2, 3]. Die Mitglieder des Netzwerk Kindersimulation e. V. und des PAEDSIM e.V. - sowie die damit assoziierten medizinischen Einrichtungen haben in den letzten Jahren zahlreiche simulationsbasierte inner- und außerklinische Kindernotfalltrainings durchgeführt und wissen deshalb sehr gut um die Notwendigkeit und den allgemeinen Nutzen dieser Trainings. Dies ist v. a. jenen Mitarbeiter*innen im Gesundheitswesen bewusst, die alltäglich kritische Situationen in der Kinder- und Jugendheilkunde bewältigen müssen.

Die durch das „severe acute respiratory syndrome coronavirus 2" (SARSCoV-2) ausgelöste Pandemie stellt uns alle vor gewaltige gesellschaftliche und medizinische Herausforderungen, und vieles, was uns in Zukunft erwarten wird, ist aktuell nicht vorhersehbar. Sehr

Die Autoren schreiben im Namen des Netzwerk Kindersimulation e. V.und von PAEDSIM e.V. wahrscheinlich ist aber, dass wir alle unsere beruflichen Gewohnheiten und Routinen ändern werden müssen, auch was die Durchführung von Aus- und Fortbildungen im medizinischen Bereich betrifft. Während einige Formate online bzw. digital - z. B. als Webinar oder E-Simulation - abgehalten werden können, gilt dies für die simulationsbasierten (Team-)Trainings nur sehr bedingt. Eine der bedeutendsten Stärken simulationsbasierter Teamtrainings stellt in den aktuellen Zeiten deren größte Herausforderung dar: Sie baut entscheidend auf im Szenario und unter Stress spontan ablaufenden Teaminteraktionen und -entscheidungen auf. Realitätsnah durch Teaminteraktion zustande kommende (Be-)Handlungsprozesse und deren Bewertung im nachfolgenden „debriefing" sind jene Schlüsselkomponenten, die die didaktische Überlegenheit dieser Teamtrainings gegenüber anderen Lehrmethoden ausmachen. Demzufolge ist unserer Meinung nach „echte LivePatienten-Simulation“ als Methode des Teamtrainings nicht durch Onlineformate ersetzbar, sondern kann durch diese nur ergänzt werden. Virtuelle, computerbasierte Simulationen und Telesimulationsformate ermöglichen in gewissen Bereichen alternatives simula- tionsbasiertes Lernen, jedoch sind diese Formate technisch und logistisch mitunter aufwendig und haben im deutschsprachigen Raum bisher nur in geringem Umfang Verbreitung gefunden (z.B. im Rahmen des Annual Congress 2020 der European Society for Emergency Medicine [EUSEM], 19.-22.09.2020, www. eusem.org).

Da bei der Versorgung eines pädiatrischen Notfalls im Simulationstraining - wie im klinischen Alltag auch - die Wahrung eines adäquaten Sicherheitsabstands nur schwerlich möglich ist, sind in den letzten Monaten sehr viele dieser Trainings völlig zu Recht und medizinisch begründet abgesagt worden. Von Institutionen, wie z.B. dem Österreichischen Rat für Wiederbelebung, gibt es mittlerweile jedoch Stellungnahmen, die eine Wiederaufnahme strukturierter Notfallkurse unter strengen Sicherheitsauflagen und klar definierten Rahmenbedingungen empfehlen [4]. Für die Patientensimulation an pädiatrischen Kliniken und Gesundheitseinrichtungen stellt sich unserer Meinung nach jedoch die Frage, wie gut solche Empfehlungen zur Abstandwahrung und zur räumlichen Entzerrung mit der Wiederaufnahme essenzieller Teamtrainings vereinbar sind, da die- 
se eine gewisse Zahl an im Szenario handelnden und miteinander direkt interagierenden Personen nebst zumindest ein bis zwei erfahrenen Trainer ${ }^{*}$ innen erfordern. Um unseren Patient ${ }^{*}$ innen mehr anstatt weniger Sicherheit zu bieten, wird es wie vor der SARS-CoV-2Pandemie erforderlich sein, das gesamte Team einer Klinik, Abteilung oder Station wieder regelmäßig zu trainieren. Es gilt daher abzuwägen, ob das mögliche, mit entsprechenden hygienischen Maßnahmen wohl geringe Risiko eines lokalen Infektion-Cluster jenes eines schlecht trainierten Behandlungsteams aufwiegt oder nicht.

Maßnahmen wie regelmäßige Händeund Equipment-Desinfektion, das dauerhafte Tragen von Mund-Nasen-Schutz, die Einhaltung der Husten- und Niesetikette sowie - wo möglich - die Wahrung des Mindestabstands sollten obligate Bestandteile von Trainings in diesen Zeiten sein. Das Tragen von „Filtering-facepiece-2“(FFP2)-Masken zur Verringerung einer möglichen Ansteckungsgefahr oder die SARS-CoV-2-Testung (v.a. der externen Trainer*innen) vor und nach dem Training kann diskutiert werden, um die Sicherheit der trainierten Teams (und letztendlich der Patient ${ }^{*}$ innen) zu gewährleisten. Zusätzlich sollte in aktuellen Simulationstrainings eine Adaptation der Kursinhalte mit Integration von Notfällen, die eine Assoziation zur „coronavirus disease 2019“ (COVID-19) aufweisen, erfolgen. Gerade hier bringt die Form des Simulationstrainings den großen Vorteil mit sich, die notwenigen Abläufe bei Infektionsverdacht (z. B. Triage, Reanimation, Notfallintubation) trainieren zu können und somit die Mitarbeiterund Patientensicherheit potenziell zu verbessern.

Bei allem medizinischen Verständnis für das Pausieren simulationsgestützter Notfalltrainings im Team fragen wir uns aktuell: „Wie geht es weiter?" oder besser noch: „Wie geht es sicher weiter?“ Wie oben erläutert, fällt es uns als Ärzten und Simulationstrainern schwer, eine klare und ausschließliche Empfehlung für die Wiederaufnahme des regulären Trainingsbetriebs zu geben oder diese gar zu fordern. Auf der anderen Sei- te sind wir als Netzwerk Kindersimulation e.V. und PAEDSIM e.V. zunehmend um die gute und effektive reale Patient ${ }^{*}$ innenversorgung und damit um die Sicherheit der uns anvertrauten kleinen und größeren Patient* ${ }^{\star}$ innen besorgt. Uns treibt die Frage um, inwieweit nichtstattfindende Trainings die Behandlung realer kritisch kranker Kinder und Jugendlicher negativ beeinflussen.

Ein nächster Schritt muss deshalb sein, interprofessionell und fachgesellschaftsübergreifend einen evidenzbasierten, belastbaren Rahmen zu erstellen, mit dessen Vorgaben wir es schaffen können, die wahrscheinlich einer noch länger bestehenden Gefährdungslage durch SARSCoV-2 oder mögliche andere Erreger zu meistern, zum Wohle der uns anvertrauten Kinder!

\section{Korrespondenzadresse}

\section{Dr. Philipp Jung}

Klinik für Kinder- und Jugendmedizin, Universitätsklinikum Schleswig-Holstein, Campus Lübeck

Ratzeburger Allee 160, 23538 Lübeck, Deutschland

philipp.jung@uksh.de

Danksagung. Wir danken den Vorstandsmitgliedern des Netzwerk Kindersimulation e. V. (Anja Große-Lordemann, Annika Paulun, Katharina Bibl, Thomas Werner und Ruth Löllgen) und den Vorstandsmitgliedern von PAEDSIM e. V. (Anke Helleken, Julia Keil und Fabian Behret) für die Unterstützung und Mitwirkung an dieser Stellungnahme.

\section{Einhaltung ethischer Richtlinien}

Interessenkonflikt. P. Jung ist 1. Vorsitzender von PAEDSIM e. V. und führt für PAEDSIM und die SIMCharacters Training GmbH (Wien, Österreich) Simulationstrainings auf Honorarbasis durch. L. Mileder ist 1. Vorsitzender des Netzwerk Kindersimulation e. V. sowie stellvertretender Leiter des Clinical Skills Center und stellvertretender organisatorischer Leiter der postgraduellen Universitätslehrgänge „Medical Simulation" an der Medizinischen Universität Graz. Er führt für die SIMCharacters Training GmbH (Wien, Österreich) auf Honorarbasis simulationsbasierte Trainings durch. F. Hoffmann führt als Trainer für PAEDSIM e. V. und die SIMCharacters Training GmbH (Wien, Österreich) Simulationstrainings auf Honorarbasis durch. E. Heimberg ist als Trainerin für PAEDSIM e. V. tätig und Mitglied des Beirats des Netzwerk Kindersimulation e. V. Sie führt für PAEDSIM e. V. und die SIMCharacters Training GmbH (Wien, Österreich) Simulationstrainings auf Honorarbasis durch. Ansonsten bestehen keine finanziellen Interessenkonflikte.
Für diesen Beitrag wurden von den Autoren keine Studien an Menschen oder Tieren durchgeführt. Für die aufgeführten Studien gelten die jeweils dort angegebenen ethischen Richtlinien.

\section{Literatur}

1. Schmutz J, Manser T (2013) Do team processes really have an effect on clinical performance? A systematic literature review. $\mathrm{Br} J$ Anaesth 110(4):529-544

2. Zeng J, Qian S, Zheng M, Wang Y, Zhou G, Wang H (2013) The epidemiology and resuscitation effects of cardiopulmonary arrest among hospitalized children and adolescents in Beijing: an observational study. Resuscitation 84(12):1685-1690

3. Riva B, Clavenna A, Cartabia M, Bortolotti A, Fortino I, Merlino L, Biondi A, Bonati M (2018) Emergency department use by paediatric patients in Lombardy Region, Italy: a population study. BMJ Paediatr Open 2(1):e247

4. Schwindt J, Pflanzl-Knizacek L, Nierscher FJ, Hoffmann P, Singraber E, Cardona F, Burda G, Koestenberger M, Egger A, Schlieber J, Baubin M (2020) Durchführung von Kursen zur Aus- und Fortbildung während der COVID-19-Pandemie. www.arc.or.at/covid/.Zugegriffen:29. Juni 2020 